\title{
Fortalecimiento de las capacidades de resiliencia mediante la academia, caso de estudio: Programa de Liderazgo y Resiliencia ante Desastres, Honduras 2019
}

\section{Resumen}

De acuerdo al Panel Intergubernamental de Cambio Climático la resiliencia se define como: "la capacidad de los sistemas sociales, económicos y ambientales de afrontar un suceso, tendencia o perturbación peligrosos respondiendo o reorganizándose de modo que mantengan su función esencial, su identidad y su estructura, y conservando al mismo tiempo la capacidad de adaptación, aprendizaje y transformación" (IPCC, 2018). Por otro lado, el liderazgo es definido por Hunter (1996:38) como el "arte" de influir sobre la gente para que trabaje con entusiasmo en la consecución de un objetivo en pro del bien común; es así, que este Programa se concibe con el objetivo de fomentar el liderazgo para la resiliencia frente a desastres, dado que en los momentos de atención a emergencias, como en todos los procesos de recuperación se pone en evidencia que los lideres deberán procurar ese entusiasmo en las comunidades que les permita poder recuperarse y crear resiliencia.

Este artículo busca resumir las acciones desarrolladas y los resultados alcanzados mediante la ejecución del Programa de Liderazgo y Resiliencia ante Desastres que fue impulsado por la Universidad de George Washington y el Institute for for Disaster \& Fragility Resilience (IDFR) a través del Instituto Hondureño de Ciencias de la Tierra; en él, encontrarán detalladamente los objetivos que se plantearon, la metodología desarrollada para su alcance y los resultados obtenidos. Cabe mencionar que este Programa es un ejemplo de las acciones de vinculación desde la academia hacia la sociedad, haciendo aportes en formación de capacidades a través de la educación no formal y apoyando el proceso de rediseño curricular del Posgrado en Gestión del Riesgo y Manejo de Desastres de la UNAH.

Palabras Claves: liderazgo, resiliencia, desastres, formación de capacidades, capacidad de respuesta, educación no formal.
Autora:

Lidia E.

Torres Bernhard ${ }^{1}$ 


\section{Strengthening resilience capacities through academia, case study: Leadership and Disaster Resilience Program, Honduras 2019}

\section{SUMMARY}

According to the Intergovernmental Panel on Climate Change, resilience is defined as: "the capacity of social, economic and environmental systems to face a dangerous event, trend or disturbance, responding or reorganizing in such a way that they maintain their essential function, identity and structure, and at the same time preserving the capacity for adaptation, learning and transformation" (IPCC,2018). On the other hand, leadership is defined by Hunter (1996:38) as the "art" of influencing people to work with enthusiasm in the achievement of a goal for the common good; Thus, this Program is conceived with the objective of fostering leadership for resilience in the face of disasters, given that in times of attention to emergencies, as in all recovery processes, it is evident that leaders should seek that enthusiasm in communities that allow them to recover and build resilience. This article seeks to summarize the actions developed and the results achieved through the execution of the Leadership and Disaster Resilience Program that was promoted by the George Washington University and the Institute for Disaster \& Fragility Resilience (IDFR) through the Honduran Institute of earth Sciences; in it, you will find in detail the objectives that were set, the methodology developed for their scope and the results obtained. It is worth mentioning that this Program is an example of the linkage actions from the academy to society, making contributions in capacity building through non-educational and supporting the process of curricular redesign of the Postgraduate Program in Risk Management and Disaster Management at UNAH.

KEYWORDS: Leadership, resilience, disasters, capacity building, response capacity, non-formal education.

\section{Introducción}

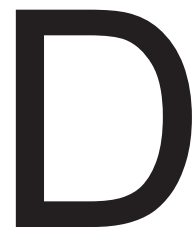

urante 2018 y 2019 , el Instituto Hondureño de Ciencias de la Tierra (IHCIT) y el Instituto y la Universidad de George Washington a través del Institute for for Disaster \& Fragility Resilience (IDFR), entablaron relaciones con el pro- pósito de que Honduras formase parte del Programa de Liderazgo y Resiliencia conocido por sus siglas en inglés como DRL. Este Programa fue financiado por la Fundación Bill \& Melinda Gates; surgió como respuesta al evidente fracaso del liderazgo observado durante y des- 
pués del huracán Katrina en 2005. El huracán Katrina fue un huracán de categoría 5 que tocó tierra en Florida y Luisiana; de acuerdo con la Red Interamericana de Mitigación de Desastres (RIMD), causó daños materiales por 75 mil millones de dólares estadounidenses, convirtiéndose en el huracán más costoso y mortífero en la historia de los Estados Unidos; la tormenta causó la muerte a 1.836 personas.

Durante los últimos 10 años, con el apoyo de la Fundación Bill y Melinda Gates (BMGF), el Fondo Mundial para la RRD del Banco Mundial (GFDRR) y el Gobierno Real de Noruega, el Instituto para la Resiliencia ante Desastres y Fragilidad (IDFR) ha promovido el programa DRL para abordar las brechas de liderazgo y abordar las causas fundamentales de la vulnerabilidad en las comunidades de todo el mundo (IDFR/DRL Report, 2019); es así que en 2018, Honduras a través del IHCIT se integra al Programa que tenía ya influencia en Centroamérica a través de El Salvador y Guatemala.

El objetivo primordial del Programa DRL se enmarca en desarrollar capacidades locales para hacer frente a las causas fundamentales de la vulnerabilidad en todos los niveles del sistema, haciendo énfasis en las organizaciones de sociedad civil. Al inicio de su accionar, el Programa se dedicó a la investigación para comprender o identificar las barreras que impiden un cambio transformador y la sostenibilidad, identificando que el liderazgo es un componente faltante en estas acciones, se identificó además que existen carencias en cuanto a programas de capacitación y/o educación para acortar esta brecha existente entre la generación de resiliencia y el liderazgo para la resiliencia frente a los desastres. En función de ello, surge la iniciativa de asocios institucionales clave entre países vulnerables en el mundo, para emprender el esfuerzo de desarrollar y fortalecer capacidades de liderazgo a nivel individual, institucional y de sistema. Así surge el Programa que ha tenido influencia en regiones como Centroamérica, el África y el Oriente Medio.

El Programa de Liderazgo y Resiliencia, resultó entonces en una iniciativa que promueve la asociación de instituciones sólidas como las universidades locales, los organismos de sociedad civil y los entes rectores de la Gestión del Riesgo en los diferentes países miembro, buscando la producción rápida y sostenida de liderazgo y redes globales de aprendizaje compartido, dando origen entre otras cosas, a la Red Global de Líderes capacitados, comprometidos a salvar vidas, aliviar el sufrimiento y reducir los costos económicos de las pérdidas y las afectaciones sociales como la 


\section{Materiales y métodos}

Dado que el enfoque del Programa DRL se dirige a los actores locales, priorizando el conocimiento y la formación de capacidades; utiliza la plataforma académica para liderar dichos procesos de capacitación, garantizando con ello la relevancia y la calidad de los contenidos y habilidades que se busca desarrollar en el individuo; lo que a su vez le permitirá fortalecer y empoderar tanto a la institución que representa como a las poblaciones afectadas. El programa Liderazgo en Resiliencia ante Desastres (DRL) busca:

1. Construir capacidad humana en las comunidades vulnerables a los desastres.
2. Salvar vidas de manera más eficaz.

3. Aliviar el sufrimiento mediante el fortalecimiento sistemático de la capacidad existente, promoviendo el desarrollo de futuros líderes liderazgos.

4. Trabajar hacia la sostenibilidad a través de una red global de académicos, actores del sector público y los actores privados.

Para ello, el Programa se diseña y se enmarca en tres componentes estratégicos o niveles estratégicos. La Figura 1, muestra los niveles estratégicos del programa y su breve definición.

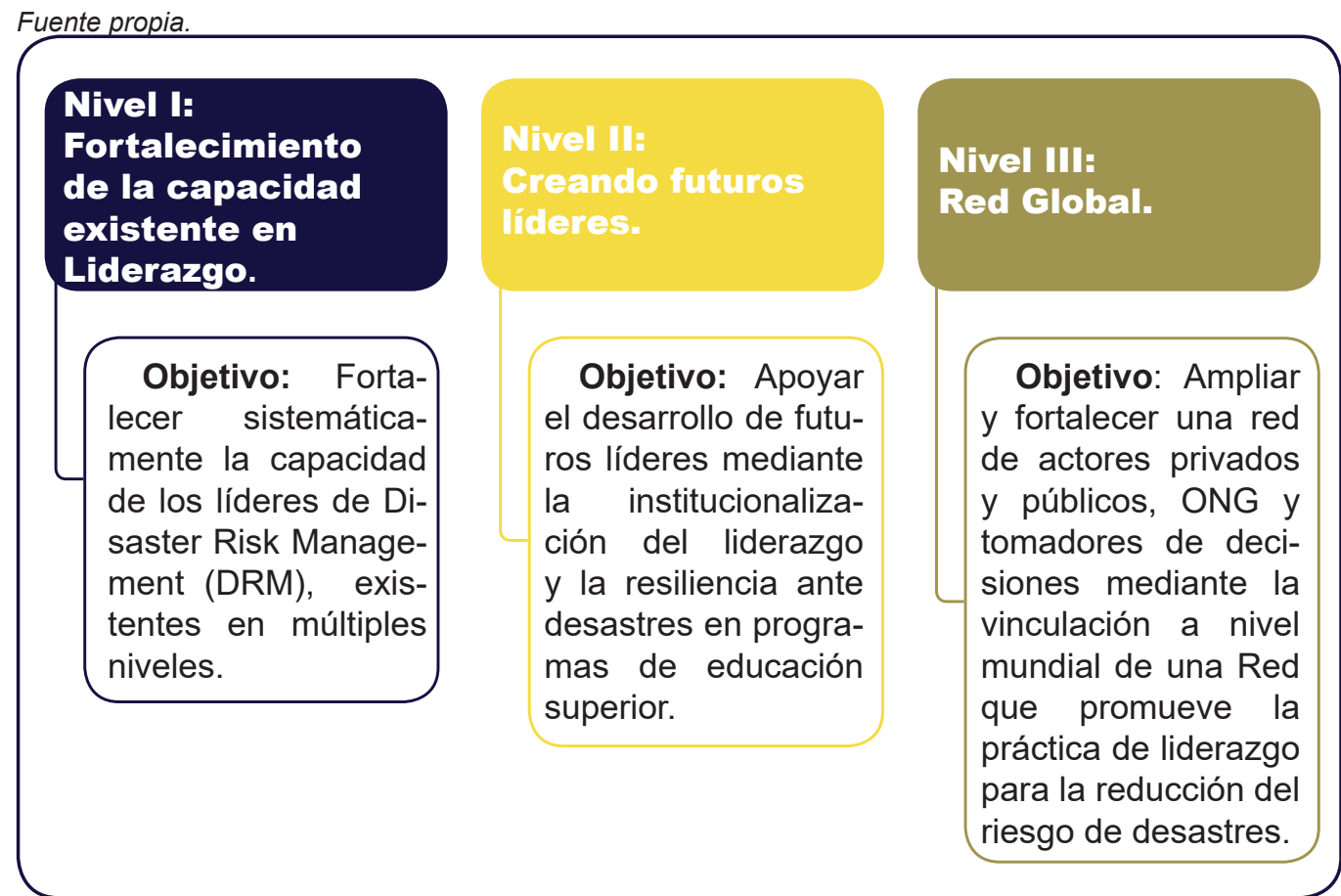

Figura 1. Niveles Estratégicos del Programa de Liderazgo y Resiliencia Honduras 2018-2019. 


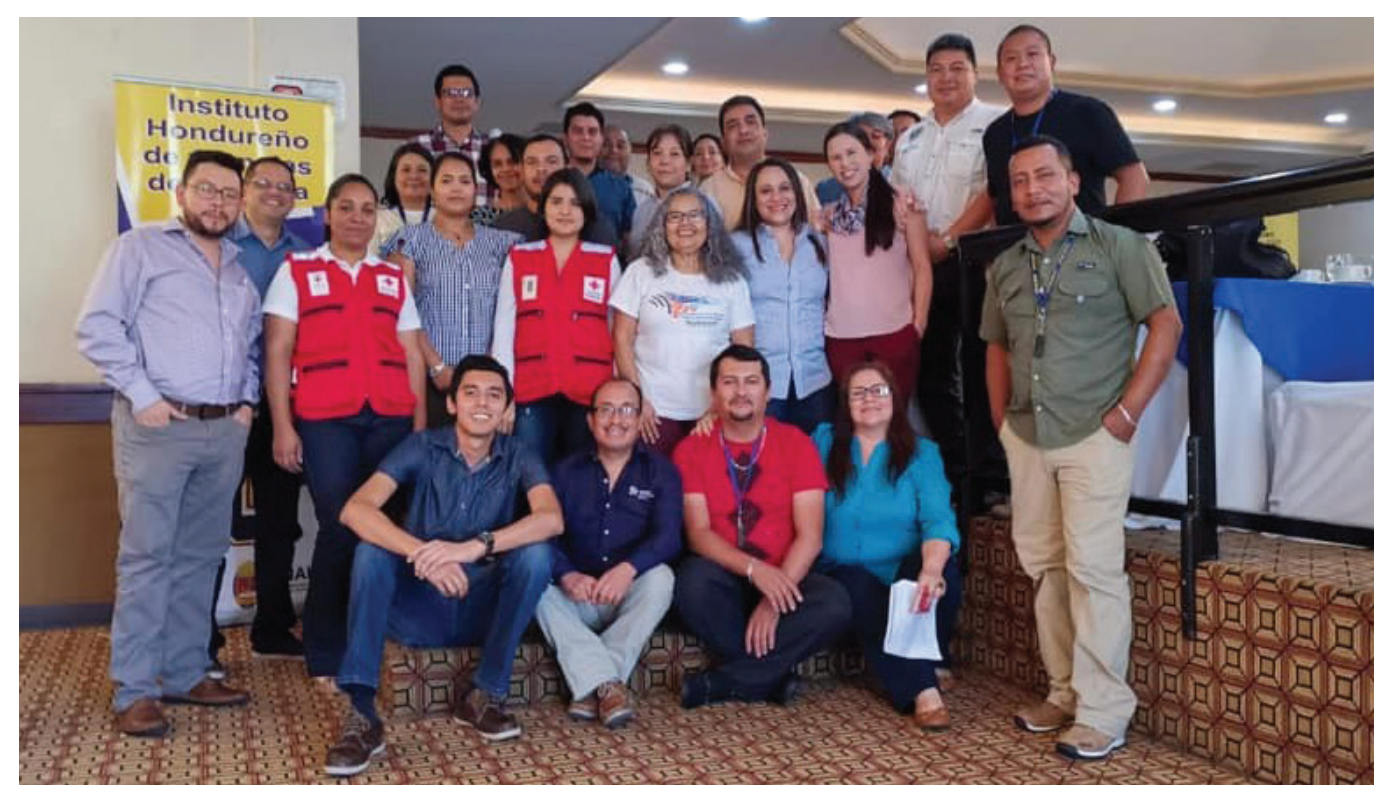

Figura 2. Grupo de Becarios Curso DRL. Programa de Liderazgo y Resiliencia IHCIT, 2019.

\section{Nivel I: El fortalecimiento de la capacidad existente de \\ Liderazgo.}

Se definen como cursos ejecutivos del Programa DRL a través del cual se gestionan becas completas para aquellos participantes que resultasen elegidos durante el proceso de selección establecido; estos cursos tienen como objetivo fortalecer la capacidad existente entre los líderes actuales que representan a todos los sectores pertinentes en la Gestión para la Reducción del Riesgo de Desastres a nivel local, nacional y regional. El Programa de Nivel 1 requiere un compromiso de ocho a doce meses duración de cada miembro del Programa DRL. En el caso de Honduras, se diseñó un Curso Virtual de Liderazgo y Resiliencia ante Desastres, impartido para hondureños y guatemaltecos de diferentes instituciones, tuvo una duración de siete meses y 10 dos encuentros presenciales.

Se dividió en dos etapas la Fase 1 , diseñada para estandarizar y fortalecer conocimientos de Gestión del Riesgo, Resiliencia y Capacidad de Respuesta y la Fase 2, se desarrolló principalmente con un enfoque de empoderar el liderazgo a nivel personal pero también el liderazgo para la Gestión de la Reducción del Riesgo y el aumento de la Resiliencia.

El curso incluyó dos sesiones presenciales de una semana de duración cada una, llamados respectivamente DRL-1 y DRL-2. Los pilares principales incluyen: Operaciones en Situaciones de Desastres, Factores Humanos, Análisis y Riesgos Ambientales.

Como parte del Programa Ejecutivo, los becarios DRL tuvieron la tarea de llevar a cabo en una evaluación práctica, aplicada en sus entornos laborales o comunidades 
de incidencia para sus respectivas organizaciones.

Además, los Becarios del Programa DRL también se unirán a la Red Global de DRL en conformada por todas la generaciones de becarios que hasta la fecha se han formado en diferentes partes del mundo.

\section{Nivel II: Creando futuros líde- res en DRL}

El nivel II del Programa DRL busca construir una masa crítica de liderazgo en resiliencia ante desastres mediante el desarrollo de capacidades facultativas, y el establecimiento o mejora de certificados de posgrado y programas de grado.

La UNAH organizó una serie de actividades encaminadas a incluir la temática de liderazgo en resiliencia frente a desastres dentro del currículo de los posgrados que se coordinan por investigadores adscritos al IHCIT; adaptando el contenido de asignaturas identificadas como ejes transversales en donde se puede incluir los tópicos liderazgo y resiliencia ante desastres con énfasis en el liderazgo de la resiliencia ante desastres, asegurando así el fortalecimiento de capacidades de los futuros líderes y promoviendo la inclusión de la temática en los programas de educación superior a nivel de país. Para ello, el comité asesor ejecutivo del Programa DRL, miembros selectos de profesionales con experiencia, profesores, investigadores, organismos gubernamentales y no gubernamentales formaron un grupo focal dirigido por Consultor(a) con vasta experiencia que lideró la transversalización de DRL en los contenidos de las asignaturas seleccionadas dentro de la Maestría en Gestión del Riesgo y Manejo de Desastres de la Escuela de Física en la Facultad de Ciencias de la UNAH; este proceso ha sido un aporte para el rediseño curricular del posgrado en mención, y será implementado en la siguiente cohorte (Figura 3).

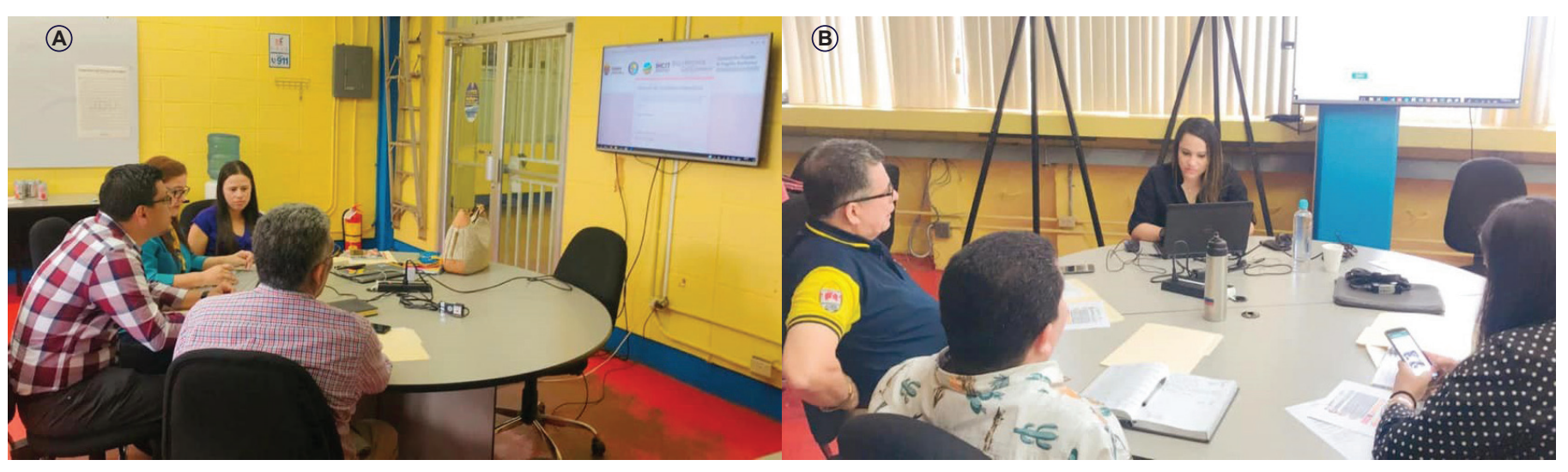

Figura 3. (A) y (B) Talleres de revisión del pensum de Maestría en Gestión del Riesgo y Manejo de Desastres para la inversión de la temática de liderazgo y resiliencia en espacios curriculares pertinentes. 


\section{Nivel III - Red Global DRL}

En un esfuerzo por continuar el desarrollo de una comunidad de aprendizaje DRL, se crean plataformas sistemáticas para el intercambio de conocimientos entre las organizaciones mediante el aprovechamiento de los recursos educativos abiertos (REAS) compartidas por la red. En concreto, el Coordinador de la Red en DRL por parte del IHCIT/UNAH desempeña un papel decisivo en los esfuerzos para promover el uso de la red, fomentando activamente el debate y la conversación entre sus pares y DRL compañero a través de varios mecanismos innovadores incluyendo, pero no limitando a la difusión de las oportunidades regionales, las mejores prácticas, y nuevos cuestionamientos en asuntos relacionados con desastres a través del espacio de colaboración DRL.

\section{Resultados}

Para el caso de la región centroamericana, el Programa DRL tuvo incidencia en Guatemala, El Salvador, Honduras y Nicaragua; convirtiéndose en una iniciativa regional acompañada en todo momento por la Universidad de George Washington y la Fundación Bill \& Melinda Gates, para el Nivel I los países debieron trabajar en parejas, siendo para Honduras el trabajo conjunto con Guatemala. Los principales resultados observados para el Programa en general liderado por Honduras de enlistan a continuación: (a) Curso de liderazgo y resiliencia ante desastres (b) Incorporación de la Temática DRL en espacios de aprendizaje identificados dentro de la Maestría en Gestión del Riesgo y Manejo de Desastres (c) Talleres de capacitación en DRL para otras unidades académicas de la
UNAH (d) Diseño de un curso libre en gestión integrada del riesgo con enfoque de liderazgo y resiliencia ante desastres dentro del sistema virtual de la UNAH (e) ampliar y fortalecer la Red Global de Lideres frente a los desastres, a continuación se presentan los principales resultados del Programa, desarrollado en Honduras.

\section{Resultado 1: Curso de}

\section{Liderazgo y Resiliencia ante} Desastres, Honduras 2019.

Diseñado en modalidad virtual, con dos encuentros semanales presenciales y el grupo meta fueron estudiantes provenientes de Guatemala y Honduras. El diagrama que muestra la Figura 4, nos resume el proceso de implementación y los principales hallazgos de los participantes. 


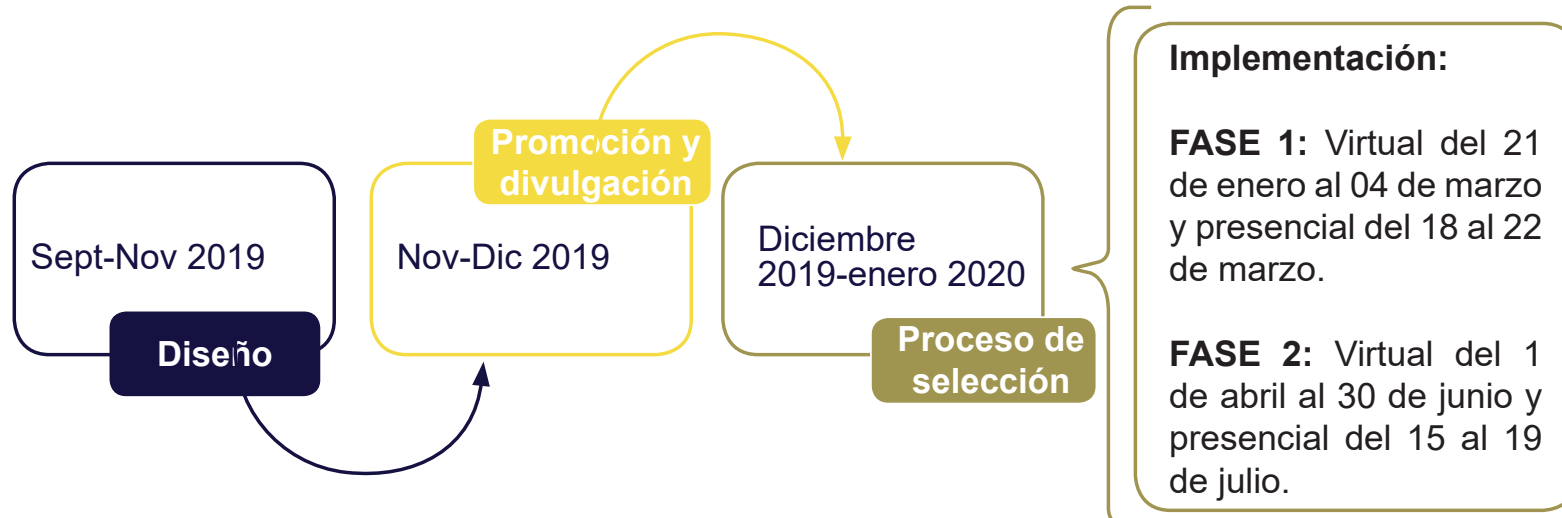

Figura 4. Fases de implementación del Nivel 1: Curso de liderazgo y resiliencia ante desastres, Honduras 2019. Instituto Hondureño de Ciencias de la Tierra.

La Fase 1 virtual del curso, se diseñó de manera tal que se pudiesen estandarizar los conocimientos de todos los participantes; en esta la fase se tocaron temas como: Componentes de la gestión del riesgo, generalidades sobre amenazas naturales recurrentes en la región centroamericana, vulnerabilidad y métodos de análisis, gestión de riesgo y el ciclo de los desastres. La Fase 1 presencial se orientó a desarrollar las temáticas de resiliencia y liderazgo aplicados a la gestión integral del riesgo, comunidades resilientes, coordinación interinstitucional y la integración de los enfoques de resiliencia y liderazgo en las formas de trabajo, la comunicación del riesgo, el rol de la educación formal y no formal para la creación de sociedades resilientes, reducción de desastres y desarrollo sostenible, enfoque de género, liderazgo y resiliencia; además, se realizaron prácticas para la presentación y análisis de casos de estudio.

La Fase 2 virtual, se enfocó en desarrollar temas como: inteligencia emocional, dimensiones de la inteligencia, IQ y $E Q$, resiliencia personal: locus de control interno y locus de control externo, huella personal-ADKAR e introspección. En la etapa presencial de esta fase se desarrollaron los estilos de liderazgo, estilo de toma de decisiones y estilos de liderazgo situacional, junto con trabajos prácticos para fomentar el liderazgo individual y de grupo, fortaleciendo y potenciando las virtudes de líderes identificados en los becarios.

Finalmente los becarios se unieron en grupos para diseñar e implementar un plan de acción que les permitiese desarrollar las habilidades y conocimientos adquiridos, los resultados alcanzados estadísticamente, se muestran en la Figura 5 (A), se observa la cantidad de estudiantes que postularon como becarios, la cantidad que fue acep- 
Fuente propia.

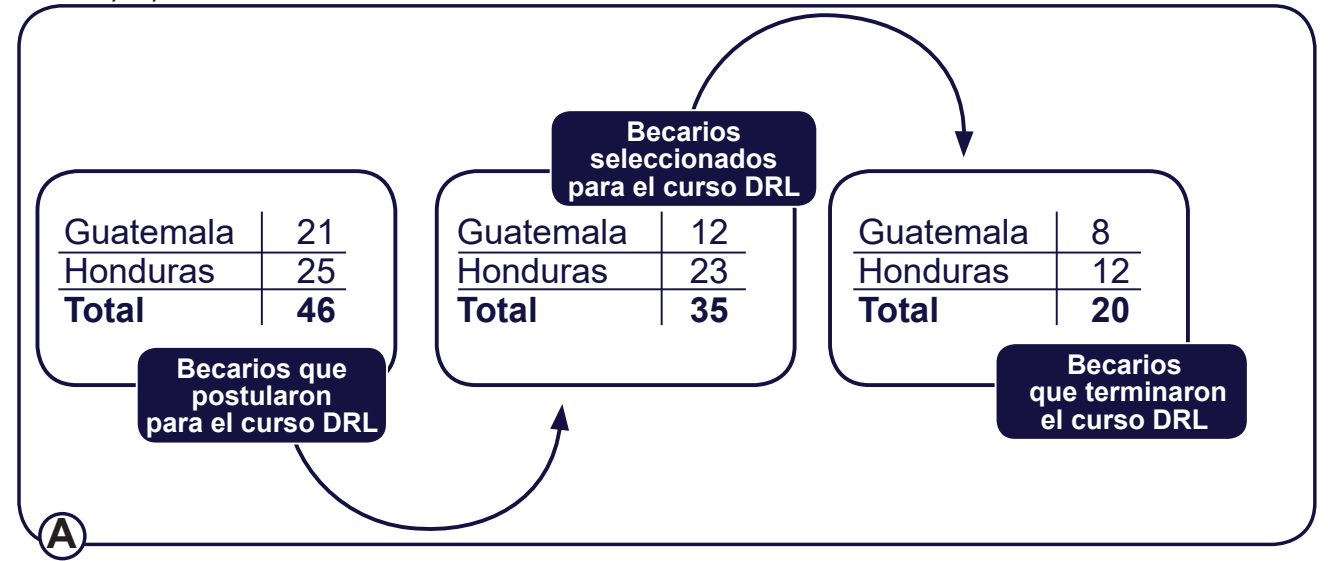

\begin{tabular}{|l|r|}
\hline \multicolumn{2}{|c|}{ Perfíl de los Becarios } \\
\hline Sector & $\%$ \\
\hline Academia & $59 \%$ \\
\hline Sociedad Civil & $19 \%$ \\
\hline Gobierno & $19 \%$ \\
\hline Sector Privado & $3 \%$ \\
\hline Género & \\
\hline Femenino & $43 \%$ \\
\hline Masculino & $57 \%$ \\
\hline
\end{tabular}

Figura 5. (A) Indicadores de participación en el Curso de liderazgo y resiliencia ante desastres, Honduras 2019. (B) Resumen de Sectores participantes y porcentaje de participación por género. Instituto Hondureño de Ciencias de la Tierra.

tada y la cantidad de estudiantes que finalizaron con éxito tanto para Honduras como para Guatemala, logrando una eficiencia terminal del $60 \%$ aproximadamente; considerado por la coordinación como aceptable, en función de la barrera que supone la virtualización y la presencialidad parcial en coordinación de participantes de dos países, en la Figura 5 (B) se presenta el resumen de incidencia por sector y por género.

\section{Resultado 2: Creando futuros líderes en DRL}

Con el objetivo de crear una masa crítica de liderazgo para la resiliencia ante desastres, mediante el desarrollo de capacidades facultativas y el establecimiento de mejoras en los certificados de posgrado, se define el segundo resultado como un aporte al proceso de re-diseño curricular de la Maestría en Gestión del Riesgo y Manejo de Desastres, incorporando en algunos espacios curriculares los tópicos de liderazgo y resiliencia a modo de transversalizar el tema.

Las Instituciones de Educación Superior (IES) tienen un rol fundamental en los esfuerzos para alcanzar los Objetivos del DesarroIlo Sostenible (ODS) (Aguiló Pons, 2017) y el aumento de la resiliencia ante desastres y el cambio climático porque son espacios de formación, sensibilización y promoción de valores que tienen el potencial de ser traducidos en cambios dirigidos hacia el desarrollo y la mejora de calidad de vida (ídem). EI ODS número cuatro "Educación de Calidad" identifica a la educación como un canal para proveer herramientas que permitan dar soluciones a los problemas que la humanidad enfrenta (ONU, 2015). Es por ello, que el IHCIT decide fortalecer el Programa de Maestría antes mencionado para introducir los tópicos de liderazgo y resiliencia en algunas asignaturas pertinentes. 
La ejecución del proyecto se realizó a través de siete actividades principales:

- Revisión del Plan de Estudio y el diseño metodológico del proceso de inserción.

- Identificación de los aprendizajes adecuados para la inserción de las temáticas de DRL.

- Adaptación de los sílabos de los espacios de aprendizajes seleccionados.

- Dos talleres de revisión para adecuar y realizar mejoras en el proceso de inserción según la opinión de expertos miembros del Comite Asesor Ejecutivo del Programa DRL (CAE).

- Elaboración del informe final que reúne los productos y resultados del proyecto.

El proceso metodológico desarrollado para el proyecto consistió en una revisión bibliográfica de tres temas: (1) metodologías existentes de procesos de inserción en el sector educativo, (2) estado del arte de la temática de resiliencia ante desastres y (3) las conexiones sobre la temática de liderazgo y la RRD. El análisis de literatura se sistematizó a través de tablas para la propuesta de contenidos generales y específicos que permitieran la selección de los espacios de aprendizaje a modificar.
Como resultado de la revisión del Programa de la MGRMD se seleccionaron cuatro cursos, o espacios de aprendizaje, que se consideraron adecuados para la inserción de los bloques temáticos de DRL. Los cursos seleccionados son: Planificación Urbana, Aspectos Socioeconómicos de los Desastres, Gestión del Riesgo y Manejo de emergencias. La Figura 6 muestra en detalle los temas que se han incluido en cada asignatura o curso.

Para facilitar la actualización de los sílabos, se elaboraron fichas de inserción para cada uno de los temas a insertar, el contenido de las fichas lleva de la descripción general, los objetivos y literatura para cada uno de los temas. Se puede decir que de este proceso se obtuvo un resultado satisfactorio $y$ que será puesto en marcha en la siguiente cohorte del Posgrado.

\section{Resultado 3: Red Global}

Su objetivo es ampliar y fortalecer una red de actores privados y públicos, ONG y tomadores de decisiones mediante la vinculación a nivel mundial de una Red que promueve la práctica de liderazgo frente a los desastres, en un esfuerzo por continuar el desarrollo de una comunidad de aprendizaje, se establecen las conexiones y contactos para el intercambio de conocimientos entre las organizaciones mediante el aprovechamiento de los recursos 


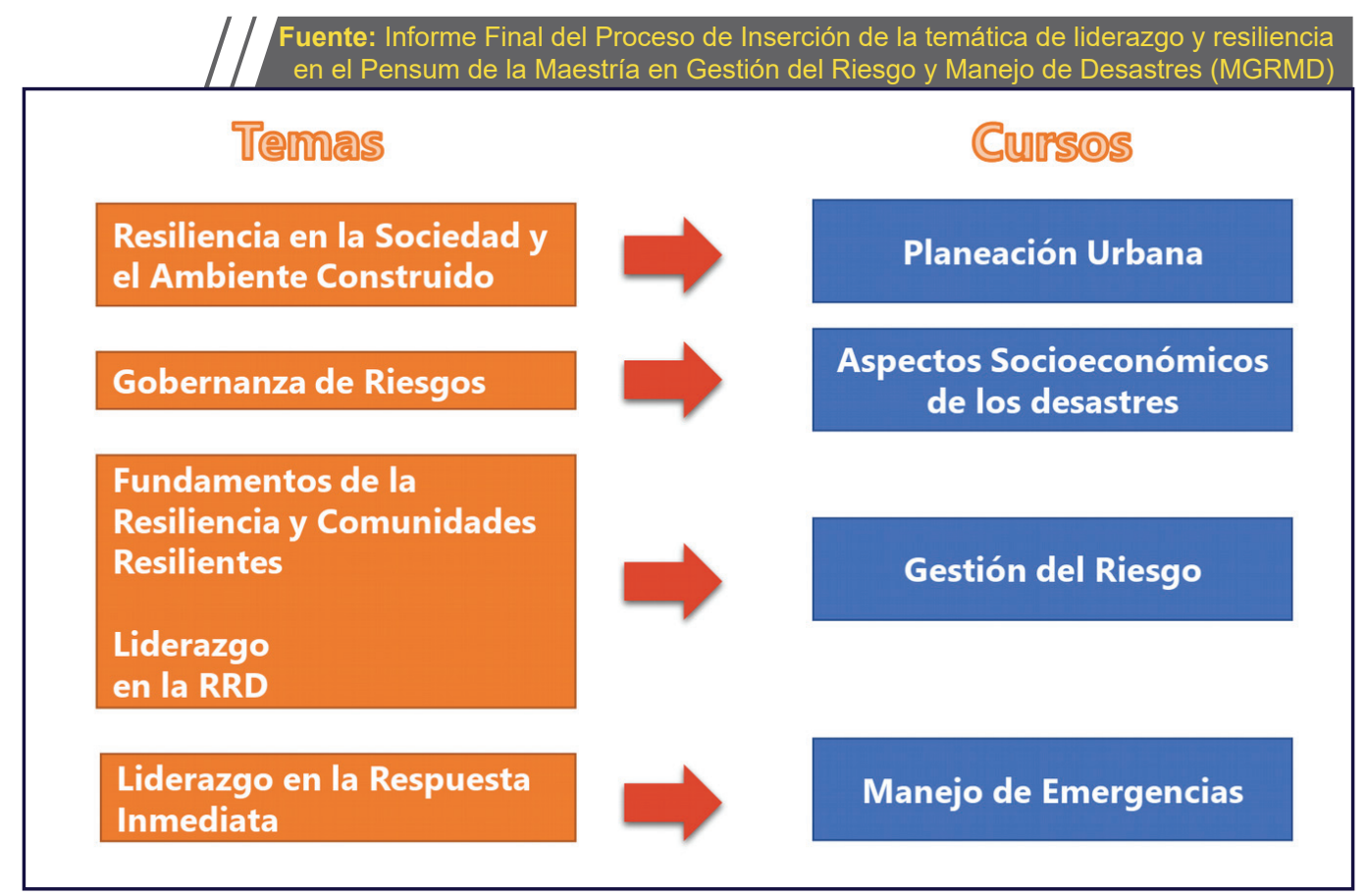

Figura 6. Cursos seleccionados para inserción de temática de DRL y bloques a ser insertados en cada uno.

educativos abiertos (REAS).

Se desarrolló en enero de 2019 un Foro Mundial de Liderazgo y Resiliencia ante Desastres, el cual tuvo lugar en Ciudad del Cabo, Sudáfrica; y contó con la presencia de países de Centroamérica y el Caribe, África, Medio Oriente y Asia. Actualmente miembros de la red se mantienen en contacto $y$ continúan promoviendo acciones, gestionando proyectos, publicaciones y procesos de divulgación hacia la sociedad.

\section{Discusión}

De la intervención antes descrita, en el marco del Programa de Liderazgo y Resiliencia y basados en los resultados alcanzados, podemos decir que desde la academia bajo el eje de vinculación, se logra formar capacidades en diferentes áreas del desarrollo de potencial humano de una nación; la educación no formal, permite un abanico de opciones para hacer incidencia en sectores sensibles para el desa16 rrollo de los países. Cabe destacar, que dentro del IHCIT esta ha sido la primera experiencia abordando el tema de liderazgo y cómo este se vuelve imprescindible para la correcta atención de las emergencias o situaciones de desastre, además para la creación de capacidades orientadas a la resiliencia. Un punto clave a destacar, es que el liderazgo es una virtud humana que se pone a prueba en diferentes situaciones 
del diario vivir; sin embargo las personas que están desarrollándose profesionalmente para la atención a emergencias, para salvaguardar la vida humana como fin primordial de la gestión de riesgos y reducción de desastres, deben autoevaluarse, hacer introspección y encontrar dentro de si esas características de un líder y debe saber explotarlas para obtener ese cambio en las unidades sociales que debe intervenir. Sin duda alguna, esta experiencia ha dejado una muy buena percepción en los involucrados que fueron formados bajo el Curso de Liderazgo y Resiliencia ante Desastres, pero también ha supuesto una fortaleza inconmensurable para poder ofrecer a la sociedad una masa crítica de profesionales a nivel superior que se verán beneficiados en su haber del conocimiento a través del re-diseño de la Maestría en Gestión del Riesgo y Manejo de Desastres; quienes, en su próxima promoción, recibirán conocimiento de estos tópicos dentro de su pensum académico. Aquí la impresión de una de las participantes del Curso DRL, como ex- becaria del Programa en Honduras:

"La segunda fase del Curso DRL, fortaleció la inteligencia emocional adentrándonos en nuestros perfiles de liderazgo, el contexto del curso me condujo para incrementar mi red profesional incorporando a mis compatriotas, mis compañeros guatemaltecos y los socios de Nicaragua y El Salvador sobre todo en los encuentros presenciales; incluyendo también al personal de IHCIT-UNAH, maestros y asesores que facilitaron la formación del DRL y los enlaces con la Universidad de George Washington y la Fundación Gates". - Aurora Genoveva González Muñoz, Becaria de Honduras 2019. Voluntario del Consejo Nacional de Impacto para la Gestión de Riesgos (MNIGR). 


\section{Conclusiones}

El programa DRL logró abordar las brechas de capacidad existentes en el liderazgo. Esto se logró a través de cultivar y fomentar la asociación interinstitucional sólida con universidades locales y el aprovechamiento de estos motores de cambio existentes para la producción rápida y sostenida de las facultades de liderazgo mediante redes globales de aprendizaje compartido.

Durante el Programa se gestionaron herramientas que servirán de manera práctica en la vida profesional pero también personal de los becarios del Curso DRL, fomentando con ello el liderazgo y potenciando las características que cada individuo posee para ejercerlo.

La virtualización supone un reto, pero también una enorme oportunidad para llevar el conocimiento a través de las barreras territoriales, esto permitió que grupos de estudiantes de Guatemala y Honduras, pudiesen compartir a lo largo de siete meses entre ellos pero también con sus instructoras desde Brasil y Sudáfrica. Este tipo de interacción es muy enriquecedora y finalmente los encuentros presenciales, se convierten en el espacio de fortalecer la empatía y el trabajo en equipo.

Los procesos de rediseño curricular en los posgrados son de carácter mandatorio, puesto que en un mundo cambiante la necesidad de la actualización impera y se debe adoptar para poder dar respuesta a las necesidades de la sociedad actual; con esto la Universidad cumple su compromiso con Honduras de ofrecer programas académicos en educación formal como no formal que fomenten el desarrollo sostenible.

Algunos retos deberán enfrentarse de cara al futuro y entre ellos podemos mencionar, la virtualización y puesta en oferta de este tipo de cursos a través de la plataforma virtual de la UNAH, dar seguimiento al Programa ya no de la mano del ente promotor sino de manera auto-sostenible y poder mantener viva la iniciativa en el tiempo. La vinculación hacia la sociedad es la cuerda que le da valor a las acciones desde la academia y es por ello que es importante que estos procesos de formación lleven consigo el desarrollo de Planes de Acción que fortalezcan las comunidades. 


\section{Referencias bibliograficas}

Aguiló Pons, A. (2017). El rol crucial de las universidades ante la Agenda Global del Desarrollo Sostenible. En VIII Congreso Iberoamericano de Universidades Promotoras de la Salud. Universidad de Alicante. 29-29 junio. Disponible en: https://web.ua.es/es/congreso-iupsalud-alicante2017/ documentos/sintesis-conferencias/conferencia-3-antoni-aguilo.pdf

Hunter, J.C. (1996). La Paradoja. Un relato sobre la verdad esencial del liderazgo. Barcelona: Empresa Activa.

IPCC, 2018. Anexo I: Glosario [Matthews J.B.R. (ed.)]. En: Calentamiento global de $1,5^{\circ} \mathrm{C}$, Informe especial del IPCC sobre los impactos del calentamiento global de $1,5^{\circ} \mathrm{C}$ con respecto a los niveles preindustriales y las trayectorias correspondientes que deberían seguir las emisiones mundiales de gases de efecto invernadero, en el contexto del reforzamiento de la respuesta mundial a la amenaza del cambio climático, el desarrollo sostenible y los esfuerzos por erradicar la pobreza [Masson-Delmotte V., P. Zhai, H.-O. Pörtner, D. Roberts, J. Skea, P.R. Shukla, A. Pirani, W. Moufouma-Okia, C. Péan, R. Pidcock, S. Connors, J.B.R. Matthews, Y. Chen, X. Zhou, M.I. Gomis, E. Lonnoy, T. Maycock, M. Tignor y T. Waterfield (eds.)].

Ky Luu, 2019. IDFR/DRL Reporte de Retroalimentación del Programa de Liderazgo y Resiliencia.

ONU. (2015). Educación de Calidad: Por qué es importante. Disponible en: https://www.un.org/sustainabledevelopment/es/wpcontent/uploads/ sites/3/2016/10/4_Spanish_Why_it_Matters.pdf

RIMD, 2009. Red Interamericana de Mitigación de Desastres. Documento: Huracán Katrina, cuatro años después (El Porvenir). 29 de agosto de 2009. http://www.rimd.org/documento.php?id=446 